\title{
Systematic tracking of disrupted modules identifies significant genes and pathways in hepatocellular carcinoma
}

\author{
MENG-HUI ZHANG ${ }^{1}$, QIN-HAI SHEN ${ }^{2}$, ZHAO-MIN QIN ${ }^{3}$, QIAO-LING WANG ${ }^{4}$ and XI CHEN ${ }^{5}$ \\ ${ }^{1}$ Department of General Surgery, The Fourth Hospital of Jinan, Jinan, Shandong 250031; Departments of ${ }^{2}$ Medicine and \\ ${ }^{3}$ Nursing, Shandong Medical College, Jinan, Shandong 250002; ${ }^{4}$ Department of Ophthalmology, The Second Hospital of Jinan, \\ Jinan, Shandong 250022; ${ }^{5}$ Department of Ophthalmology, The Ninth Hospital of Chongqing, Chongqing 400700, P.R. China
}

Received May 6, 2015; Accepted July 12, 2016

DOI: $10.3892 / 01.2016 .5039$

\begin{abstract}
The objective of the present study is to identify significant genes and pathways associated with hepatocellular carcinoma (HCC) by systematically tracking the dysregulated modules of re-weighted protein-protein interaction (PPI) networks. Firstly, normal and HCC PPI networks were inferred and re-weighted based on Pearson correlation coefficient. Next, modules in the PPI networks were explored by a clique-merging algorithm, and disrupted modules were identified utilizing a maximum weight bipartite matching in non-increasing order. Then, the gene compositions of the disrupted modules were studied and compared with differentially expressed (DE) genes, and pathway enrichment analysis for these genes was performed based on Expression Analysis Systematic Explorer. Finally, validations of significant genes in HCC were conducted using reverse transcription-quantitative polymerase chain reaction (RT-qPCR) analysis. The present study evaluated 394 disrupted module pairs, which comprised 236 dysregulated genes. When the dysregulated genes were compared with $211 \mathrm{DE}$ genes, a total of 26 common genes [including phospholipase $\mathrm{C}$ beta 1 , cytochrome $\mathrm{P} 450(C Y P) 2 \mathrm{C} 8$ and $C Y P 2 B 6]$ were obtained. Furthermore, 6 of these 26 common genes were validated by RT-qPCR. Pathway enrichment analysis of dysregulated genes demonstrated that neuroactive ligand-receptor interaction, purine and drug metabolism, and metabolism of xenobiotics mediated by CYP were significantly disrupted pathways. In conclusion, the present study greatly improved the understanding of HCC in a systematic manner and provided potential biomarkers for early detection and novel therapeutic methods.
\end{abstract}

Correspondence to: Dr Qin-Hai Shen, Department of Medicine, Shandong Medical College, 5460 South Second Ring Road, Jinan, Shandong 250002, P.R. China

E-mail: qinhaishenmedi@yeah.net

Key words: hepatocellular carcinoma, modules, protein-protein interaction network, dysregulated gene, pathway, reverse transcription-quantitative polymerase chain reaction

\section{Introduction}

Hepatocellular carcinoma (HCC) is the fifth most common cancer worldwide and the third leading cause of cancer-associated mortality (1), which explains the importance of identifying novel early diagnostic markers and therapeutic targets (2). HCC primarily develops from cirrhosis caused by chronic infection with hepatitis B virus or hepatitis $\mathrm{C}$ virus (HCV), alcoholic injury, and, to a lesser extent, from genetically determined disorders (3). However, the heterogeneity of HCC presents unique challenges in identifying biomarkers and exploring molecular pathogenesis in this disease (4).

Identifying genes that are differentially expressed (DE), exhibit similar expression profiles to known disease genes, are 'central' or 'reachable' in disease molecular networks, or display disease associations according to the literature is the main method to evaluate biomarkers (5). In addition, a crucial distinguishing factor of cancer genes is their involvement in core mechanisms responsible for genome stability and cell proliferation (such as DNA damage repair and cell cycle), and the fact that these genes function as highly synergetic or coordinated groups (6). Therefore, critical to implicating genes in cancer is the identification of core modules, including pathways and complexes, that are dysregulated in cancer.

Beyond straightforward scoring genes in a gene regulatory network, it is crucial to study the behavior of modules across specific conditions in a controlled manner in order to understand the disease mechanisms and to identify biomarkers (7). For example, Zhang et al (8) have identified tightly connected gene co-expression sub-networks across 30 cancer networks in various cell lines, and have tracked aberrant modules as frequent sub-networks appearing across these cancers. However, studying multiple cancers simultaneously makes it challenging to discern clearly the intricate underlying mechanisms.

Furthermore, it is important to effectively integrate omics data into such an analysis. For example, Magger et al (9) combined protein-protein interaction (PPI) and gene expression data to construct tissue-specific PPI networks for 60 tissues, and used them to prioritize disease genes. A few significant genes may not be identifiable through their own behavior, but their changes are quantifiable when considered in conjunction with other genes (which is known as modules) (10). Therefore, 
a systematic tracking of gene and module behavior across specific conditions in a controlled manner is required. Besides, since a number of human genes have not yet been assigned to definitive pathways, scoring pathways based on module analysis has become a more reliable analyzing approach compared to individual gene analysis.

Therefore, the present study systematically tracked the disrupted modules of re-weighted PPI networks to identify significant DE genes and pathways between normal controls and HCC patients, in order to reveal potential biomarkers for HCC. To achieve this, normal and HCC PPI networks were firstly inferred based on Pearson correlation coefficient (PCC). Next the modules in the PPI networks were explored based on a clique-merging algorithm, and disrupted modules were identified by matching normal and HCC modules. Subsequently, the gene compositions of the disrupted modules were studied and compared with DE genes, and pathway enrichment analysis was performed for these genes. Finally, dysregulated genes of $\mathrm{HCC}$ were validated utilizing reverse transcription-quantitative polymerase chain reaction (RT-qPCR) analysis.

\section{Materials and methods}

\section{Inferring normal and HCC PPI networks}

HumanPPInetworkconstruction.Adatasetofliterature-curated human PPIs from the Search Tool for the Retrieval of Interacting Genes/Proteins (STRING; string-db.org/), comprising 16,730 genes and 1,048,576 interactions, was utilized (11). For STRING analysis, self-loops and proteins without expression value were removed. The remaining largest connected component with score $>0.75$ was kept as the selected PPI network, which consisting of 9,273 genes and 58,617 interactions.

Gene expression dataset and dataset preprocess. The microarray expression profiles of E-GEOD-14520 $(12,13)$ from the ArrayExpress database (www.ebi.ac.uk/arrayexpress/) were selected for the study. In E-GEOD-14520, there were a total of 488 samples, which were processed on two platformed. To eliminate the batch effects, only samples processed on the GeneChip ${ }^{\circledR}$ Human Genome U133A 2.0 Array (Affymetrix, Inc., Santa Clara, CA, USA), were recruited in the present study, which consisted of 123 samples. The gene expression profiles were preprocessed with standard methods, including background correction via 'robust multiarray average (rma)' (14), 'quantiles' (15), 'mas' (16) and 'medianpolish' (14), and were subsequently screened with a feature filter method.

Briefly, in order to eliminate the influence of nonspecific hybridization, background correction was applied by the 'rma' method (14). The observed perfect match (PM) probes were modeled as the sum of a normal noise component $N$ (normal with mean $\mu$ and variance $\sigma^{2}$ ) and an exponential signal component $S$ (exponential with mean $\alpha$ ). To avoid negative values, the normal was truncated at zero. An adjustment was performed based on the observed intensity $O$ as follows:

$$
E(s \mid O=o)=a+b \frac{\phi\left(\frac{a}{b}\right)-\phi\left(\frac{o-a}{b}\right)}{\Phi\left(\frac{a}{b}\right)+\Phi\left(\frac{o-a}{b}\right)-1}
$$

, where $a=s-\mu-\sigma^{2}{ }_{\alpha}$ and $b=\sigma$. Of note, $\varnothing$ and $\Phi$ were the standard normal distribution density and distribution function, respectively. Mismatch (MM) probe intensities were corrected by 'mas'.

Normalization was performed through a quantiles-based algorithm (15). Specifically, the transformation $x_{i}^{\prime}=\mathrm{F}-{ }^{1}\left[\mathrm{G}\left(x_{i}\right)\right]$ was used, where $G$ was estimated by the empirical distribution of each array and $F$ was estimated using the empirical distribution of the averaged sample quantiles. Using the 'mas' method to conduct PM/MM correction (16), an ideal mismatch was subtracted from PM. The ideal MM would always be less than the corresponding PM, and thus, it could be safely subtracted without the risk of achieving negative values.

The summarization method was 'medianpolish' (14). A multichip linear model was fit to the data from each probe set. In particular, for a probe set $k$ with $I=1, \ldots, I_{k}$ probes and data from $j=1, \ldots, J$ arrays, the following model was fitted:

$$
\log _{2}\left(\mathrm{PM}_{i j}^{k}\right)=\alpha_{i}^{k}+\beta_{j}^{k}+\varepsilon_{i j}^{k}
$$

where $\alpha_{i}$ was a probe effect and $\beta_{j}$ was the $\log _{2}$ expression value.

Next, the data were screened by the feature filter method of the genefilter package version 1.54 .2 (bioconductor.org/packages/release/bioc/html/genefilter.html). The gene expression value for each gene was obtained, and the number of genes with multiple probes was determined to be 12,493 .

Re-weighting gene interactions by PCC. In the present study, PCC was selected to re-weight gene interactions in HCC and normal networks. PCC was a measure of the correlation between two variables, assigning a value between -1 and +1 inclusive, and evaluated the probability of two co-expressed gene pairs (17). The PCC of a pair of genes (X and Y), which encoded the corresponding paired proteins ( $u$ and $v$ ) interacting in the PPI network, was defined as:

$$
P C C(X, Y)=\frac{1}{s-1} \sum_{i=1}^{s}\left(\frac{g(X, i)-\bar{g}(X)}{\sigma(X)}\right) \cdot\left(\frac{g(Y, i)-\bar{g}(Y)}{\sigma(Y)}\right)
$$

where $s$ was the number of samples of the gene expression data; $g(X, i)$ or $g(Y, i)$ was the expression level of the gene $X$ or $Y$ in the sample $i$ under a specific condition; $\bar{g}(X)$ or $\bar{g}(Y)$ represented the mean expression level of gene $X$ or $Y$, and $s(X)$ or $s(Y)$ represented the standard deviation of the expression level of the gene $X$ or $Y$.

If the PCC for $X$ and $Y$ had a positive value, there was a positive linear correlation between $u$ and $v$. In addition, the PCC of each gene-gene interaction was defined as the weight value of the interaction. By re-weighting the interactions in the generic PPI network of normal and HCC samples, two conditional PPI networks were inferred.

Identifying modules from the PPI networks. The present study applied a clique-merging algorithm to identify modules of HCC and normal controls $(18,19)$. Finding all the maximal cliques from the weighted PPI and merging highly overlapped cliques were the two steps in the algorithm. The score of a clique $C$ was defined as its weighted interaction density $d_{W}(C)$ (20): 


$$
d_{W}(C)=\frac{\sum_{u \in C, v \in C} w(u, v)}{|C| \cdot(|C|-1)}
$$

where $w(u, v)$ was the weight of the interaction between $u$ and $v$. The cliques were ranked according to their scores, and the maximal cliques were obtained. A depth-first algorithm was utilized to enumerate all maximal cliques, and non-maximal cliques were effectively removed.

Next, a set of cliques $\left\{C_{1}, C_{2}, \ldots, C_{k}\right\}$ was ranked in descending order of their scores, and their ordered list was assessed by repeatedly merging highly overlapping cliques to constructed modules. For a clique $C_{i}$, it was estimated whether $C_{j}(j>i)$ existed, such that the overlap $\left|C_{i} \cap C_{j}\right| /\left|C_{j}\right|$ was $\geq t$. If $C_{j}$ existed, weighted inter-connectivity $I_{w}$ was used to determine whether $C_{j}$ should be removed or merged with $C_{i}$. If $I_{w}\left(C_{i}, C_{j}\right)$ was $\geq m, C_{j}$ was merged with $C_{i}$; otherwise, it was removed. In this setting, $t=0.5$ and $m=0.25$ were predefined thresholds for merging (6). The $I_{w}$ between the non-overlapping genes of $C_{i}$ and $C_{j}$ was calculated as follows:

$$
I_{w}\left(C_{i}, C_{j}\right)=\sqrt{\frac{\sum_{u \in\left(C_{i}-C_{j}\right)} \sum_{v \in C_{j}} w(u, v)}{\left|C_{i}-C_{j}\right| \cdot\left|C_{j}\right|} \cdot \frac{\sum_{u \in\left(C_{j}-C_{i}\right)} \sum_{v \in C_{i}} w(u, v)}{\left|C_{j}-C_{i}\right| \cdot\left|C_{i}\right|}}
$$

Comparing modules between HCC and normal control. To identify altered modules, normal and HCC modules were matched by setting a high $t_{J}$, which ensured that the module pairs either had the same gene composition or had lost or gained only a few genes. The module sets of normal and HCC were denoted as $S=\left\{S_{1}, S_{2}, \ldots, S_{m}\right\}$ and $T=\left\{T_{1}, T_{2}, \ldots, T_{n}\right\}$, respectively. The module correlation density $d_{c}\left(S_{i}\right)$ or $d_{c}\left(T_{i}\right)$ for each $S_{i} \varepsilon S$ or $T_{i} \varepsilon T$ was calculated as follows:

$$
d_{c}\left(S_{i}\right)=\frac{\sum_{X, Y \in S_{i}} P C C((X, Y), M)}{\left|S_{i}\right| \cdot\left(\left|S_{i}\right|-1\right)}
$$

The disrupted modules $\gamma(S, T)$ were identified based on a maximum weight bipartite matching (21). The matching worked in three steps. For the first step, a similarity graph $M=\left(V_{M}, E_{M}\right)$ was built, where $V_{M}=\{S \cap T\}, E_{M}=\cap\left\{S_{i}, T_{j}\right): J(S i, T j) \geq t_{J}$ and $\Delta_{C}\left(S_{i}, T_{j}\right) \geq \delta$, whereby $J\left(S_{i}, T_{j}\right)=\left|S_{i} \cap T_{j}\right| /\left|S_{i} \mathrm{U} T_{j}\right|$ weighted every edge $\left(S_{i}, T_{j}\right)$ and was the Jaccard similarity, while $\Delta_{C}\left(S_{i}, T_{j}\right)=\left|d_{c}\left(S_{i}\right)-d_{c}\left(T_{i}\right)\right|$ was the differential correlation density between $S_{i}$ and $T_{j}$, being $t_{J}$ and $\delta$ thresholds with $2 / 3$ and 0.1 , respectively (6). In the second step, the disrupted module pairs were identified and ordered in descending sequence of their differential density $\Delta_{C}$. In the last step, genes involved in HCC were inferred as $\Gamma=\left\{g: g \in S_{i} \cap T_{j},\left(S_{i}, T_{j}\right) \varepsilon \gamma(S, T)\right\}$ ranked in non-increasing order of $\Delta_{C}\left(S_{i}, T_{j}\right)$.

Pathway enrichment analysis. The Database for Annotation, Visualization and Integrated Discovery (DAVID; david.ncifcrf. gov/) for Kyoto Encyclopedia of Genes and Genomes (KEGG; www.genome.jp/kegg/) pathway enrichment analysis was evaluated to further investigate the biological functions of the genes in the modules that were altered between the normal controls and the HCC patients (22). KEGG pathways with $\mathrm{P}<0.001$ were selected based on the Expression Analysis Systematic Explorer (EASE; david.ncifcrf.gov/ease/ease.jsp) test applied in DAVID. EASE analysis of the regulated genes indicated molecular functions and biological processes unique to each category (23). The EASE score was used to detected the significant categories. In both the functional and pathway enrichment analysis, the threshold of minimum number of genes for the corresponding term $>2$ was considered significant for a category:

$$
P=\frac{\left(\begin{array}{c}
a+b \\
a
\end{array}\right)\left(\begin{array}{c}
c+d \\
c
\end{array}\right)}{\left(\begin{array}{c}
n \\
a+c
\end{array}\right)}
$$

where $n=a^{\prime}+b+c+d$ was the number of background genes; $a^{\prime}$ was the gene number of one gene set in the gene lists; $a^{\prime}+b$ was the number of genes in the gene list including $\geq 1$ gene set; $a^{\prime}+c$ was the gene number of one gene list in the background genes; and $a^{\prime}$ was replaced with $a=a^{\prime}-1$ in EASE.

Identification of DE genes. The Linear Models for Microarray Data (www.bioconductor.org/packages/release/bioc/html/limma.html) method was used to detect DE genes between HCC patients and normal controls based on 12,493 filtered genes. The P-values for all genes were converted into the form of $-\log _{10}$ upon being manipulated with $t$ and $F$ tests. Linear fit, empirical Bayes statistics and false discovery rate correction were applied to the data by using the Fit function (bioconductor.org/packages/release/bioc/ manuals/limma/man/limma.pdf) (24). DE genes were identified for further research with a threshold of $\mathrm{P}<0.05$ and $\log _{2}$ fold-changel $>2$.

RT-qPCR analysis. RT-qPCR was used to validate the common genes of DE genes and dysregulated genes, which was explored by the gene compositions of modules and DE genes. Total RNA was prepared from 24 HCC patients using TRIzol reagent (Invitrogen; Thermo Fisher Scientific, Inc., Waltham, MA, USA). Complementary DNA was synthesized using SuperScript ${ }^{\circledR}$ Reverse Transcriptase and oligo(dT) primers (Invitrogen; Thermo Fisher Scientific, Inc.). The data were normalized to housekeeping gene $\beta$-actin, which was used as an internal reference, and relative gene expression levels were determined using the $\Delta \Delta \mathrm{Cq}$ method (25). A total of 8 common genes [including cytochrome $\mathrm{P} 450$ (CYP)2E1, CYP2C9, glutathione S-transferase Z1, CYP3A4, CYP1A2, phospholipase $\mathrm{C}$ beta 1 (PLCB1), CYP2C8 and $C Y P 2 B 6]$ were analyzed, whose primer sequences are listed in Table I.

For PCR amplification, each $4 \mu \mathrm{l}$ reaction contained $10 \mu \mathrm{l}$ of 10X PCR Buffer I (Invitrogen; Thermo Fisher Scientific, Inc.), $1 \mu \mathrm{l}$ of Taq DNA Polymerase (Invitrogen; Thermo Fisher Scientific, Inc.), $3 \mu \mathrm{l}$ of each forward and reverse primer, and $8 \mu \mathrm{l}$ of deoxynucleotides. The conditions were as follows: $2 \mathrm{~min}$ at $94^{\circ} \mathrm{C}$ for pre-denaturation, followed by 35 cycles of $10 \mathrm{sec}$ at $94^{\circ} \mathrm{C}, 15 \mathrm{sec}$ at $59^{\circ} \mathrm{C}$ and $30 \mathrm{sec}$ at $72^{\circ} \mathrm{C}$, and a final 7 -min extension at $72^{\circ} \mathrm{C}$. In total, 10 replicates of the assay within or between runs were performed to assess its reproducibility. Gene expression was examined with an $\mathrm{iCycler}_{\mathrm{iQ}}^{\mathrm{TM}}$ Real Time PCR Detection System (Bio-Rad Laboratories, Inc., Hercules, CA, USA) using an $\mathrm{iQ}^{\mathrm{TM}}$ SYBR Green PCR kit (Bio-Rad Laboratories, Inc.). 
Table I. Primer sequences for the genes validated by reverse transcription-quantitative polymerase chain reaction.

Primers (5'-3')

\begin{tabular}{lllc}
\cline { 2 - 3 } Genes & \multicolumn{1}{c}{ Forward } & \multicolumn{1}{c}{ Reverse } & Size, bp \\
\hline$P L C B 1$ & AGTCCGCCAAAAAGGACAGT & TACAAGAAAGTTGGGCACAGAG & 763 \\
$C Y P 2 C 8$ & TCTTTCACCAATTTCTCAAAAGTCT & CCAAAATTCCGCAAGGTTGTGA & 248 \\
$C Y P 2 B 6$ & AACCAGACGCCTTCAATCCT & GGGGAGTCAGAGCCATTGTC & 345 \\
$C Y P 3 A 43$ & CAAGGGATGGCACCGTAAGT & CCCCACGCCAACAGTGATTA & 586 \\
$C Y P 2 E 1$ & CTCCTCGTCATATCCATCTG & GCAGCCAATCAGAAATGTGG & 473 \\
GMNN & AAAAACGGAGAAAGGCGCTG & GTACAAGAAAGTTGGGCATATACA & 335 \\
$\beta$-actin & AAGTACTCCGTGTGGATCGG & TCAAGTTGGGGGACAAAAAG & 615
\end{tabular}

PLCB1, phospholipase C beta 1; CYP, cytochrome P450; GMNN, geminin.

A

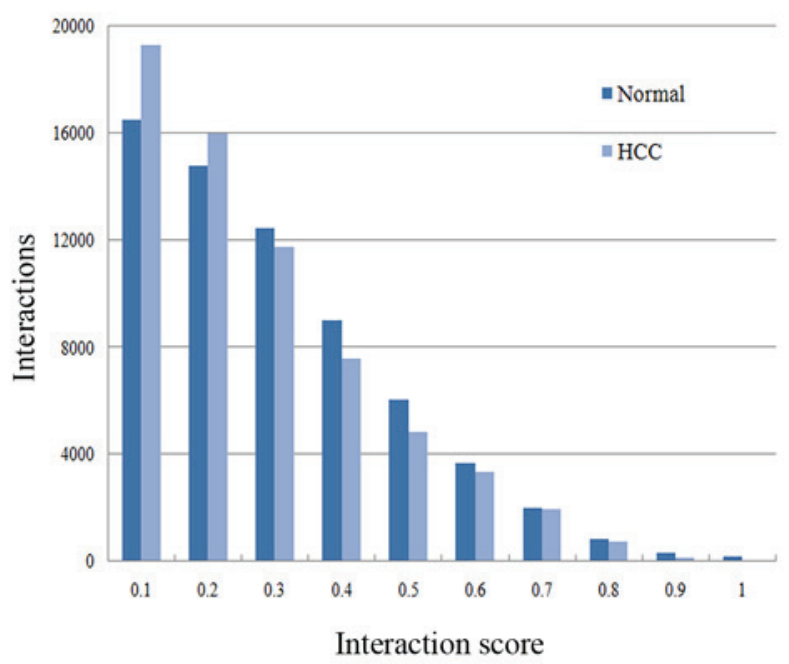

B

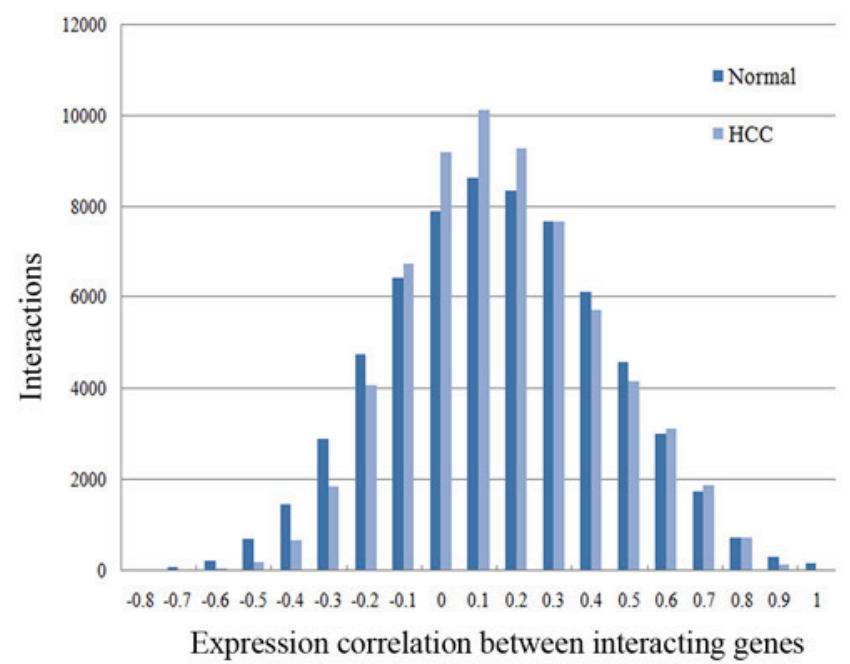

Figure 1. (A) Score-wise distribution of interactions. (B) Expression correlation-wise distribution of interactions in normal and hepatocellular carcinoma. HCC, hepatocellular carcinoma.

\section{Results}

Analyzing disruptions in HCC PPI networks. A total of DE 12,493 genes between normal and HCC individuals were obtained after preprocessing the gene expression profiles. The intersections between these gene interactions and the STRING PPI network were investigated, and PPI networks of normal and HCC individuals were identified. These networks displayed equal numbers of nodes $(7,264)$ and interactions $(45,286)$, but the interaction score or weight between two genes was different. Fig. 1 reveals that there were significant differences in the score distribution of the two networks (Fig. 1A), while the overall distribution of expression correlations between interacting genes of normal and HCC PPI networks was similar (Fig. 1B). When the correlation ranged from 0 to 0.1 , the number of interactions reached its maximum value and the interaction numbers of $\mathrm{HCC}$ were more than that of normal controls.

Analyzing disruptions in HCC modules. In the present study, a clique-merging algorithm was selected to evaluate disrupted modules between normal and HCC networks. A comparative analysis between normal $S$ and HCC $T$ modules was performed to understand these disruptions at the module level. As indicated in Table II, 1,810 and 785 modules from normal and HCC PPI networks were explored, respectively. The average module size (gene number) was 20.175 for normal and 28.851 for HCC. The maximal module correlation density among HCC modules was larger than that of normal modules, and the average correlation density of HCC modules was also larger than that of normal modules. Furthermore, the distributions of correlation density in normal and HCC modules were studied (Fig. 2). There were significant differences between normal and HCC module distribution when the correlation density ranged from -0.2 to 0.1 , and the amount of normal modules was higher than that of HCC in this range. When the correlation density was $>0.2$, the number of $\mathrm{HCC}$ modules was higher than that of normal modules. In addition, Table II also indicated an overall decrease in correlation in the HCC modules. Furthermore, this decrease had affected mainly the highly correlated modules (Fig. 2). With the thresholds $t_{J}=2 / 3$ and $\delta=0.1,394$ disrupted module pairs $[\gamma(S, T)]$, were evaluated. 
Table II. Properties of normal and HCC modules.

Module correlation density

\begin{tabular}{lccccc} 
Module set & Number of modules & Average module size & Maximal & Average & Minimum \\
\hline Normal $S$ & 1810 & 20.175 & 0.512 & 0.142 & -0.175 \\
HCC $T$ & 785 & 28.851 & 0.525 & 0.156 & -0.145
\end{tabular}

${ }^{\mathrm{a}}$ Gene number. HCC, hepatocellular carcinoma.

Table III. Correlations of matched normal and hepatocellular carcinoma module pairs.

\begin{tabular}{lcccr}
\hline & & \multicolumn{2}{c}{$\Delta_{\mathrm{c}}$} \\
\cline { 3 - 4 } Module pair subset $^{\mathrm{a}}$ & No. of pairs & Maximal & Average & Minimum \\
\hline$\gamma^{\prime}(S, T)$ & 332 & 0.215 & 0.114 & 0.100 \\
$\gamma^{\prime \prime}(S, T)$ & 62 & 0.211 & 0.123 & 0.101 \\
\hline
\end{tabular}

${ }^{a} \gamma^{\prime}(S, T) \subseteq \gamma(S, T), \gamma "(S, T) \subseteq \gamma(S, T)$.

Subsequently, $\gamma(S, T)$ were divided into $\gamma^{\prime}(S, T)$ of module pairs exhibiting higher correlation density in HCC than in normal patients, $\gamma "(S, T)$ of module pairs with lower correlation in HCC than in normal controls, and computed $\gamma^{\prime}(S, T)=332$ and $\gamma^{\prime \prime}(S, T)=62$. The absolute differential correlation density $\Delta_{C}$ of these subsets was calculated, as presented in Table III. Notably, the maximal and minimum absolute differential correlation density $\Delta_{C}$ was similar, and the difference between the average $\Delta_{C}$ of two subsets was only 0.09. Besides, pathway-based analysis revealed enrichment for similar terms in both $\gamma^{\prime}(S, T)$ and $\gamma^{\prime \prime}(S, T)$, which was not sufficiently specific to differentiate the roles of the two subsets and, therefore, whether compensatory or HCC-driving mechanisms are involved This prompted further in-depth analysis of the modules.

In-depth analysis of disrupted modules. Of the 394 disrupted module pairs in $\gamma(S, T)$, gene compositions were studied, as represented in Fig. 3. Gene compositions between two disrupted modules may be the same (Fig. 3A and B) or different (Fig. 3C and D). For the same gene compositions modules, the interaction score between two identical genes was different. For the different gene compositions modules, the genes and interaction scores were all different.

Furthermore, a total of 236 dysregulated genes were evaluated in all the disrupted modules, and pathway enrichment analysis was conducted for these genes (Table IV). There were 38 pathways within the threshold $\mathrm{P}<0.001$. Neuroactive ligand-receptor interaction $\left(\mathrm{P}=1.38 \times 10^{-22}\right)$, chemokine signaling pathway $\left(\mathrm{P}=8.22 \times 10^{-13}\right)$, metabolism of xenobiotics by CYP $\left(\mathrm{P}=8.66 \times 10^{-12}\right)$, purine metabolism $\left(\mathrm{P}=5.39 \times 10^{-11}\right)$ and linoleic acid metabolism $\left(\mathrm{P}=9.10 \times 10^{-10}\right)$ were the five most significant pathways.

If a gene existed in a normal module but not in a disrupted HCC module, it was called 'miss' gene; on the contrary, if a gene existed in a disrupted HCC module but not in a normal module, it was called 'add' gene. The present study evaluated

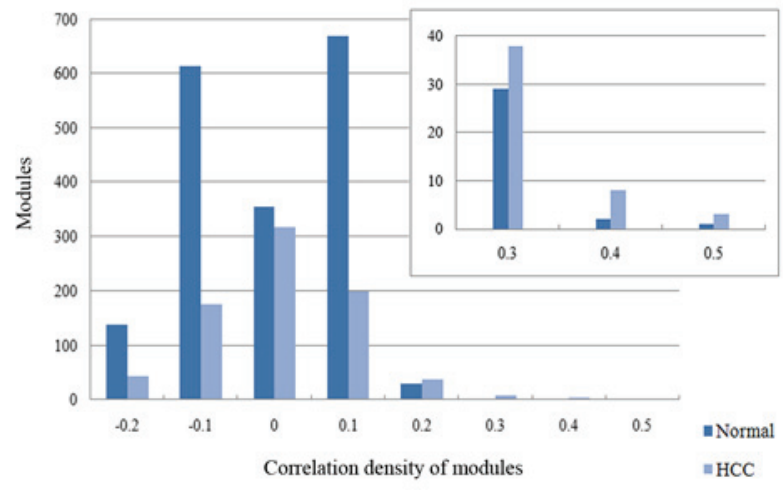

Figure 2. Distribution of correlation density of modules in normal and hepatocellular carcinoma (inset, zoom into 0.3-0.5). HCC, hepatocellular carcinoma.

58 'add' genes and 87 'miss' genes among 236 dysregulated genes of disrupted HCC modules, and collectively referred to them as dysregulated genes. The 'add' genes were enriched in two pathways (neuroactive ligand-receptor interaction and purine metabolism), while the 'miss' genes were enriched in six terms (neuroactive ligand-receptor interaction, purine metabolism, pyrimidine metabolism, chemokine signaling pathway, gap junction and RNA polymerase). Neuroactive ligand-receptor interaction and purine metabolism were common pathways of total, 'add' and 'miss' dysregulated genes enrichment analysis; thus, it was inferred that the two pathways may be significant terms in HCC development.

To explore the significant genes of HCC in depth, $211 \mathrm{DE}$ genes between normal controls and HCC patients were identified, and the intersection of DE genes and dysregulated genes was obtained. The 26 common genes are listed in Table V. PLCB1 participated in 10 pathways, of which, 7 were the same as pathways based on dysregulated genes. Platelet-derived growth factor receptor, alpha polypeptide 
A

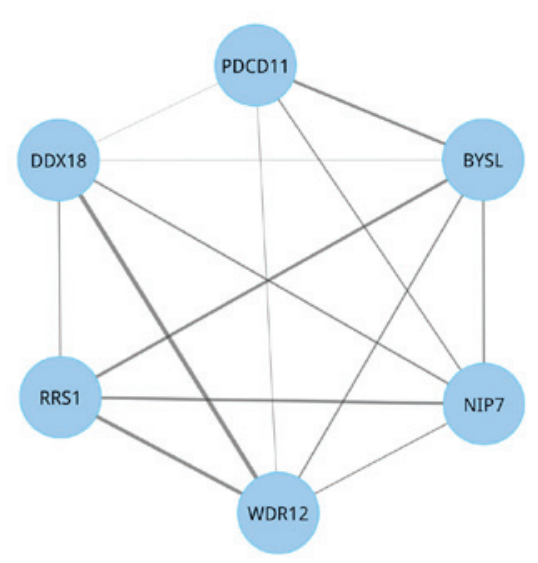

C

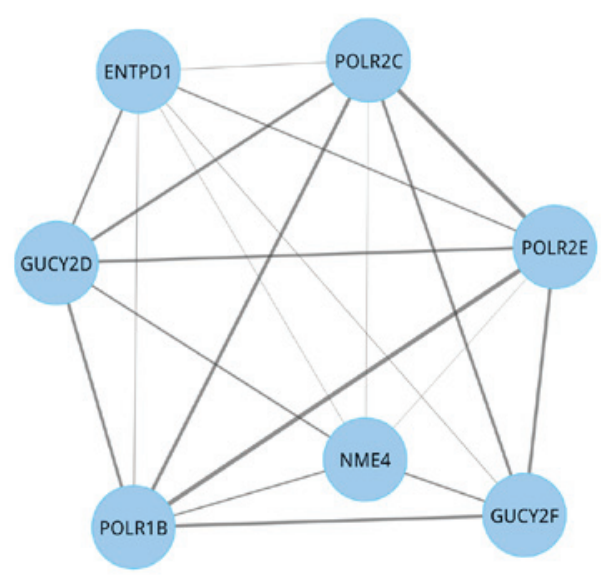

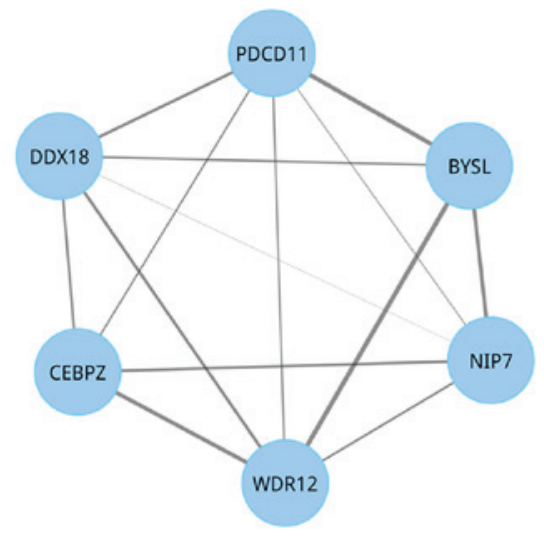

D

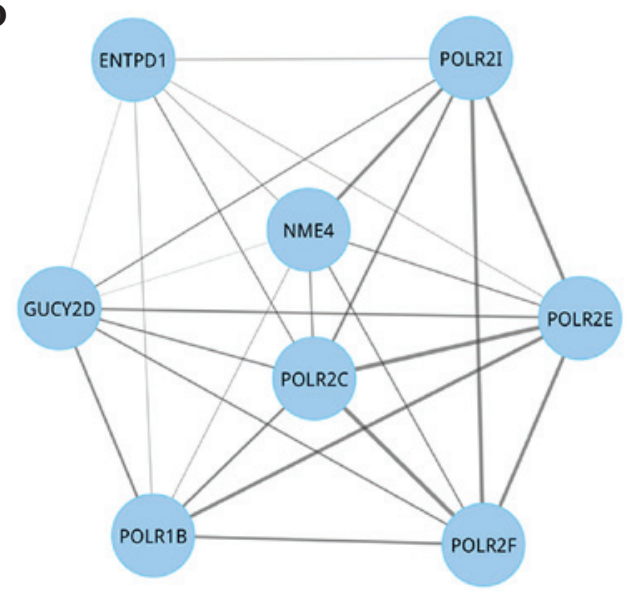

Figure 3. Swapping behavior of disrupted modules. A swapping phenomenon means that novel genes replace existing genes, forming physical interactions with the remaining ones in these modules within a tumor. (A) A module in normal condition. (B) The paired disrupted module of (A) in hepatocellular carcinoma. (C) A normal module. (D) The paired disrupted module of (C). Relative to normal conditions, a novel gene $C E B P Z$ in module (B) replaced the existing gene $R R S 1$, and in module (D) a novel gene $P O L R 2 F$ replaced the existing gene $G U C Y 2 F$. Furthermore, a new $P O L R 2 I$ gene was added. In addition, the interaction score changed. Nodes represent genes, while lines represent the interactions between these genes. The thickness of the lines represents the interaction score between two genes. PDCD11, programmed cell death 11; BYSL, bystin-like; NIP7, nucleolar pre-rRNA processing protein; WDR12, WD repeat domain 12; $R R S 1$, ribosome biogenesis regulator homolog; DDX18, DEAD box protein 18; CEBPZ, CCAAT/enhancer binding protein (C/EBP), zeta; ENTPD1, ectonucleoside triphosphate diphosphohydrolase 1; POLR2C, polymerase (RNA) II (DNA directed) polypeptide C; $N M E 4$, NME/NM23 nucleoside diphosphate kinase 4; GUCY2D, guanylate cyclase 2D; $P O L R 1 B$, polymerase (RNA) I polypeptide B; $G U C Y 2 F$, guanylate cyclase $2 \mathrm{~F}$; $P O L R 2 F$, polymerase (RNA) II (DNA directed) polypeptide F; POLR2E, polymerase (RNA) II (DNA directed) polypeptide E; POLR2I, polymerase (RNA) II (DNA directed) polypeptide I.

was involved in 9 pathways, whereas 11 common genes were not enriched in any pathway. Among these common genes, $P L C B 1, C Y P 2 C 8, C Y P 2 B 6$, alcohol dehydrogenase $(A D H) 1 \mathrm{~B}, A D H 6, C Y P 3 A 43, C Y P 2 E 1$, aurora kinase A, decorin, lipoprotein(A), geminin $(G M N N)$ and alphafetoprotein $(A F P)$, were also 'add' or 'miss' genes. The pathways of metabolism of xenobiotics mediated by CYP and drug metabolism both contained 9 common genes.

Validation of common genes based on RT-qPCR analysis. Among the common genes, PLCB1, CYP2C8, CYP2B6, $C Y P 3 A 43, C Y P 2 E 19$ and $G M N N$ were selected as examples to validate the results of the present module-based analysis and to examine the changes in their expression profiles by RT-qPCR in HCC patients (Fig. 4). These genes were also 'add' or 'miss' genes; among them, PLCB1, CYP2C8, CYP2B6, CYP2E19 and $G M N N$ were upregulated DE genes, whereas $C Y P 3 A 43$ was a downregulated DE gene. The results revealed that $P L C B 1$, CYP2C8, CYP2B6, CYP3A43, CYP2E19 and GMNN in HCC

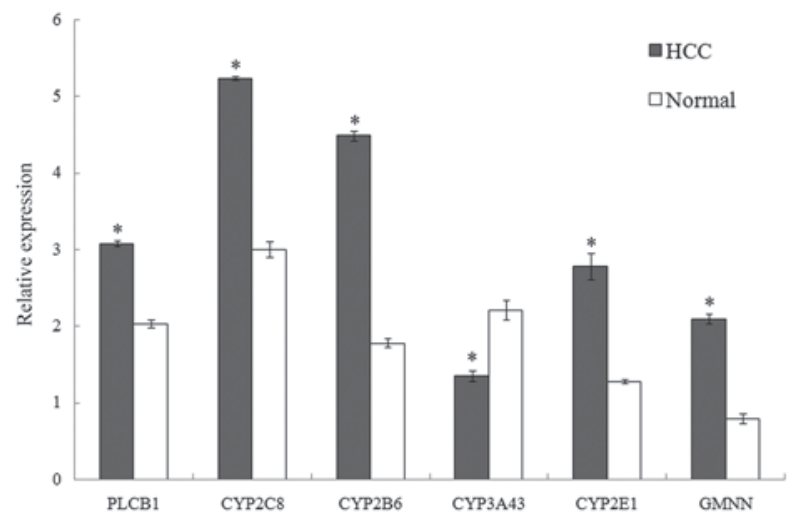

Figure 4. Relative expression of $P L C B 1, C Y P 2 C 8, C Y P 2 B 6, C Y P 3 A 43$ $C Y P 2 E 19$ and GMNN. The expression of one gene in HCC compared with normal controls was indicated by its P-value. All the six genes analyzed were significantly differently expressed in $\mathrm{HCC}\left({ }^{*} \mathrm{P}<0.001\right.$ vs. control). If a gene exhibited a $\mathrm{P}>0.05$, it would be not significantly differently expressed; by contrast, a gene with $\mathrm{P}<0.05$ was considered to be significantly differently expressed. PLCB1, phospholipase C beta 1; CYP, cytochrome P450; GMNN, geminin; HCC, hepatocellular carcinoma. 
Table IV. Pathways based on dysregulated genes in disrupted normal and hepatocellular carcinoma modules.

\begin{tabular}{|c|c|c|}
\hline Term & P-value & Count \\
\hline Neuroactive ligand-receptor interaction & $1.38 \times 10^{-22}$ & 48 \\
\hline Chemokine signaling pathway & $8.22 \times 10^{-13}$ & 31 \\
\hline Metabolism of xenobiotics by cytochrome $\mathrm{P} 450$ & $8.66 \times 10^{-12}$ & 18 \\
\hline Purine metabolism & $5.39 \times 10^{-11}$ & 26 \\
\hline Linoleic acid metabolism & $9.10 \times 10^{-10}$ & 12 \\
\hline Drug metabolism & $1.78 \times 10^{-09}$ & 16 \\
\hline Pyrimidine metabolism & $2.80 \times 10^{-09}$ & 19 \\
\hline GnRH signaling pathway & $4.74 \times 10^{-09}$ & 19 \\
\hline Phosphatidylinositol signaling system & $1.81 \times 10^{-07}$ & 15 \\
\hline VEGF signaling pathway & $2.16 \times 10^{-07}$ & 15 \\
\hline Arachidonic acid metabolism & $3.40 \times 10^{-07}$ & 13 \\
\hline Progesterone-mediated oocyte maturation & $1.24 \times 10^{-06}$ & 15 \\
\hline Vascular smooth muscle contraction & $1.31 \times 10^{-06}$ & 17 \\
\hline Inositol phosphate metabolism & $1.83 \times 10^{-06}$ & 12 \\
\hline Gap junction & $1.91 \times 10^{-06}$ & 15 \\
\hline Fc epsilon RI signaling pathway & $2.31 \times 10^{-06}$ & 14 \\
\hline Long-term depression & $3.61 \times 10^{-06}$ & 13 \\
\hline Pancreatic cancer & $5.73 \times 10^{-06}$ & 13 \\
\hline Glioma & $8.95 \times 10^{-06}$ & 12 \\
\hline Non-small cell lung cancer & $1.35 \times 10^{-05}$ & 11 \\
\hline Renal cell carcinoma & $2.54 \times 10^{-05}$ & 12 \\
\hline Melanogenesis & $3.40 \times 10^{-05}$ & 14 \\
\hline RNA polymerase & $3.63 \times 10^{-05}$ & 8 \\
\hline Fc gamma R-mediated phagocytosis & $1.01 \times 10^{-04}$ & 13 \\
\hline Cell cycle & $1.02 \times 10^{-04}$ & 15 \\
\hline Type II diabetes mellitus & $1.88 \times 10^{-04}$ & 9 \\
\hline Focal adhesion & $1.99 \times 10^{-04}$ & 19 \\
\hline DNA replication & $2.02 \times 10^{-04}$ & 8 \\
\hline Prostate cancer & $2.40 \times 10^{-04}$ & 12 \\
\hline Chronic myeloid leukemia & $2.47 \times 10^{-04}$ & 11 \\
\hline Glutathione metabolism & $2.93 \times 10^{-04}$ & 9 \\
\hline Endometrial cancer & $3.88 \times 10^{-04}$ & 9 \\
\hline Aldosterone-regulated sodium reabsorption & $4.69 \times 10^{-04}$ & 8 \\
\hline Colorectal cancer & $6.26 \times 10^{-04}$ & 11 \\
\hline Small cell lung cancer & $6.26 \times 10^{-04}$ & 11 \\
\hline Melanoma & $7.38 \times 10^{-04}$ & 10 \\
\hline ErbB signaling pathway & $8.28 \times 10^{-04}$ & 11 \\
\hline Pathways in cancer & $9.62 \times 10^{-04}$ & 24 \\
\hline
\end{tabular}

GnRH, gonadotropin-releasing hormone; Fc, fragment, crystallizable; VEGF, vascular endothelial growth factor; ErbB, erythroblastic leukemia viral oncogene.

patients were significantly $\mathrm{DE}(\mathrm{P}<0.001)$ compared with normal controls. Apart from CYP3A43, the other genes possessed higher relative expression levels in $\mathrm{HCC}$ patients than in normal controls, which confirmed the regulation of DE genes.

\section{Discussion}

The objective of the present study is to identify dysregulated genes and pathways in HCC based on systematically tracking the disrupted modules of re-weighted PPI networks. Normal and HCC PPI networks were evaluated by PCC; modules from the networks were then identified, and 394 disrupted module pairs were obtained. The gene compositions of the disrupted modules were studied, and 236 dysregulated genes of these modules were identified. When comparing these dysregulated genes with $211 \mathrm{DE}$ genes, a total of 26 common genes (including PLCB1, CYP2C8 and CYP2B6) were identified. Pathway enrichment analysis of dysregulated genes demonstrated that 
Table V. Common genes between differentially expressed genes and dysregulated genes of hepatocellular carcinoma.

\begin{tabular}{|c|c|c|c|c|}
\hline Symbol & Sum. pathway & 'Add' gene & 'Miss' gene & Pathways \\
\hline PLCB1 & 10 & No & Yes & $\begin{array}{l}\text { Long-term depression, GnRH signaling pathway, } \\
\text { gap junction, phosphatidylinositol signaling } \\
\text { system, vascular smooth muscle contraction, } \\
\text { melanogenesis, Wnt signaling pathway, inositol } \\
\text { phosphate, chemokine signaling pathway, } \\
\text { long-term potentiation }\end{array}$ \\
\hline PDGFRA & 9 & No & No & $\begin{array}{l}\text { Focal adhesion, colorectal cancer, glioma, } \\
\text { melanoma, pathways in cancer, } \\
\text { prostate cancer, gap junction, } \\
\text { MAPK signaling pathway, regulation of actin }\end{array}$ \\
\hline СYР2C9 & 5 & No & No & $\begin{array}{l}\text { Metabolism of xenobiotics by CYP, } \\
\text { drug metabolism, linoleic acid metabolism, } \\
\text { arachidonic acid metabolism, retinol metabolism }\end{array}$ \\
\hline$C Y P 2 C 8$ & 5 & Yes & No & $\begin{array}{l}\text { Metabolism of xenobiotics by CYP, } \\
\text { drug metabolism, linoleic acid metabolism, } \\
\text { arachidonic acid metabolism, retinol metabolism }\end{array}$ \\
\hline CYP1A2 & 4 & No & No & $\begin{array}{l}\text { Metabolism of xenobiotics by CYP, } \\
\text { drug metabolism, linoleic acid metabolism, } \\
\text { retinol metabolism }\end{array}$ \\
\hline CYP2B6 & 4 & Yes & Yes & $\begin{array}{l}\text { Metabolism of xenobiotics by CYP, } \\
\text { drug metabolism, linoleic acid metabolism, } \\
\text { retinol metabolism }\end{array}$ \\
\hline GSTZ1 & 4 & No & No & $\begin{array}{l}\text { Metabolism of xenobiotics by CYP, } \\
\text { drug metabolism, glutathione metabolism, } \\
\text { metabolism }\end{array}$ \\
\hline$A D H 1 B$ & 4 & Yes & No & $\begin{array}{l}\text { Metabolism of xenobiotics by CYP, } \\
\text { drug metabolism, retinol metabolism, } \\
\text { tyrosine metabolism }\end{array}$ \\
\hline ADH6 & 4 & No & Yes & $\begin{array}{l}\text { Metabolism of xenobiotics by CYP, } \\
\text { drug metabolism, retinol metabolism, } \\
\text { tyrosine metabolism }\end{array}$ \\
\hline СYРЗА4 & 4 & Yes & No & $\begin{array}{l}\text { Metabolism of xenobiotics by CYP, } \\
\text { drug metabolism, linoleic acid metabolism, } \\
\text { retinol metabolism }\end{array}$ \\
\hline$C Y P 2 E 1$ & 4 & Yes & No & $\begin{array}{l}\text { Metabolism of xenobiotics by CYP, } \\
\text { drug metabolism, linoleic acid metabolism, } \\
\text { arachidonic acid metabolism }\end{array}$ \\
\hline$A D H 1 A$ & 4 & No & No & $\begin{array}{l}\text { Metabolism of xenobiotics by CYP, } \\
\text { drug metabolism, retinol metabolism, } \\
\text { tyrosine metabolism }\end{array}$ \\
\hline$C C N B 1$ & 3 & No & No & $\begin{array}{l}\text { Cell cycle, progesterone-mediated oocyte } \\
\text { maturation, oocyte meiosis }\end{array}$ \\
\hline$A U R K A$ & 1 & No & Yes & Tyrosine metabolism \\
\hline$D C N$ & 1 & Yes & No & TGF-beta signaling pathway \\
\hline$A S P M$ & 0 & No & No & - \\
\hline$R A C G A P 1$ & 0 & No & No & - \\
\hline$L P A$ & 0 & No & Yes & - \\
\hline TOP $2 A$ & 0 & No & No & - \\
\hline CENPF & 0 & No & No & - \\
\hline$G M N N$ & 0 & Yes & Yes & - \\
\hline$C D K N 3$ & 0 & No & No & - \\
\hline NUSAP1 & 0 & No & No & - \\
\hline ECT2 & 0 & No & No & - \\
\hline $\mathrm{CTH}$ & 0 & No & No & - \\
\hline
\end{tabular}


Table V. Continued.

\begin{tabular}{lcccc}
\hline Symbol & Sum. pathway & 'Add' gene & 'Miss' gene & Pathways \\
\hline AFP & 0 & No & Yes & - \\
\hline
\end{tabular}

Sum pathway, the total number of pathways that the gene participated in. The last 11 genes in the table have not yet been assigned to any KEGG pathways, thus the corresponding pathways are not given. PLCB1, phospholipase $\mathrm{C}$ beta 1; PDGFRA, platelet derived growth factor receptor, alpha polypeptide; $C Y P$, cytochrome $\mathrm{P} 450 ; G M N N$, geminin; $G S T$, glutathione S-transferase; $A D H$, alcohol dehydrogenase; $C C N B 1$, cyclin B1; AURKA, aurora kinase A; DCN, decorin; ASPM, abnormal spindle microtubule assembly; RACGAP1, Rac GTPase-activating protein 1; $L P A$, lipoprotein(A); TOP2A, topoisomerase 2-alpha; $C E N P F$, centromere protein-F; GMNN, geminin; $C D K N 3$, cyclin-dependent kinase inhibitor 3; NUSAP1, nucleolar and spindle associated protein 1; ECT2, epithelial cell transforming 2; CTH, cystathionine gamma-lyase; $A F P$, alpha-fetoprotein; GnRH, gonadotropin-releasing hormone; MAPK, mitogen-activated protein kinase; TGF, transforming growth factor.

neuroactive ligand-receptor interaction, purine metabolism, metabolism of xenobiotics by CYP and drug metabolism were significantly disrupted pathways. In addition, the expression of various common genes was validated by RT-qPCR.

$P L C B 1$ encodes an enzyme that generates the intracellular second messenger diacylglycerol inositol 1,4,5-trisphosphate from phosphatidylinositol 4,5-bisphosphate (26). PLCB1 has been validated as a carcinogen of HCC $(27,28)$. Jia et al $(27)$ suggested that $P L C B 1$ was a critical driver gene with causal roles in carcinogenesis, and may have an important role in HCC pathogenesis. HCC with recurrence exhibited enrichment of upregulated genes mapping to signaling or disease pathways associated with cell cycle regulators, including genes that encode proteins involved in the molecular mechanisms of cancer control such as PLCB1 (28). The present study identified $P L C B 1$ as an upregulated DE gene, and then validated it by RT-qPCR, which was consistent with the literature (28).

$C Y P 2 C 8$ and $C Y P 2 B 6$ both encode a member of the CYP family of enzymes (29). These enzymes are known to metabolize certain xenobiotics, including the anticonvulsive drug mephenytoin and the anticancer drugs cyclophosphamide and ifosphamide (30). The members of the CYP family, which are involved in a myriad of biological processes, were noticed to be frequently dysregulated in numerous diseases, including liver, prostate and breast cancer as well as leukemia $(31,32)$. Furthermore, a numbers of studies have revealed that $C Y P 2 C 8$ correlated with several cancers, including breast cancer (32), ovarian cancer (33) and colorectal cancer (34). Therefore, it could be inferred that $C Y P 2 C 8$ was closely associated with cancer. In addition, $C Y P 2 C 8$ was primarily distributed in the liver, and is the second most abundant member of the CYP2C family expressed in this organ (35). Zhang et al (36) suggested that $C Y P 2 C 8$ was post-transcriptionally regulated by microRNAs in human liver. $C Y P 2 C 8$ was identified as a major CYP responsible for morphine $\mathrm{N}$-demethylation in liver microsomes (37). In the present study, CYP2C8 was demonstrated to be an upregulated DE gene, and its expression was validated by RT-qPCR. The results were consistent with those from previous studies, which confirmed the feasibility and veracity of the present analysis. Therefore. it was concluded that $C Y P 2 C 8$ played a carcinogenic role in $\mathrm{HCC}$.

CYP2B6 was an 'overlooked' $\mathrm{P} 450$ isozyme, which has now been recognized to be important for xenobiotic metabolism (38). The expression of $C Y P 2 B 6$ has been investigated in a few carcinomas, including breast cancer and HCC (39). Lee et al (40) demonstrated that several single-nucleotide polymorphisms in the vascular endothelial growth factor, insulin-like growth factor 2 and $C Y P 2 B 6$ genes, which are relevant to tumor angiogenesis or drug metabolism, predisposed to the development of treatment-associated toxicity in Korean HCC patients. Furthermore, CYP2B6 expression was decreased with liver diseases progressed to the end-stage (41). Genome-wide transcriptome analysis in three pairs of non-tumorous livers/HCCs clarified that $C Y P 2 B 6$ messenger RNA displayed the lowest expression level in group $G_{3}$ with $\mathrm{G}_{1}-\mathrm{G}_{6}$ classification (42). In the present study, it was observed that $C Y P 2 B 6$ was an upregulated $\mathrm{DE}$ gene, and when validated by RT-qPCR, its relative expression level in HCC was higher than that in normal controls. Therefore, CYP2B6 had a close association with HCC.

Pathway enrichment analysis revealed the most significant pathways in the disrupted modules, including neuroactive ligand-receptor interaction, purine metabolism, metabolism of xenobiotics by CYP and drug metabolism. It has been demonstrated by Liu et al (43) that neuroactive ligand-receptor interaction and purine metabolism were both associated with $\mathrm{HCC}$, since genes expressed in human liver were involved in neuroactive ligand-receptor interaction pathways (31). AFP secreted cluster of HCC was involved in disease mutation without neuroactive ligand-receptor interaction and in cell surface receptor-linked signal transduction (44). In addition, Zhao et al (45) revealed that neuroactive ligand-receptor interaction was present in the early-, middle- and late-stages of HCC. Therefore, this pathway (neuroactive ligand-receptor interaction) appears to be important in HCC progression.

Understanding the mechanism involved in metabolic regulation has important implications in both biotechnology and medicine. It has been estimated that $\geq 1 / 3$ of all serious health problems are caused by metabolic disorders (46). The present study revealed that HCC was correlated with several metabolic pathways, including purine metabolism and metabolism of xenobiotics by CYP. Purine metabolism may serve as the salvage pathway in HCC, as suggested by the upregulation of hypoxanthine, and the results reflected metabolic responses to surgical operation in HCC patients (47). In addition, the altered purine metabolism pathway provided a promising methodology to distinguish cirrhotic $\mathrm{HCV}$ patients who were at high risk of developing HCC from those who had already progressed to HCC (48). The metabolism 
of xenobiotics by CYP is a typical liver-function-specific pathway and is important in HCC $(49,50)$. Apart from genes involved in metabolic pathways, the present study has explored common genes, including $C Y P 2 C 8, C Y P 2 B 6$, CYP3A43 and CYP2E1, which encode members of the CYP family. CYPs are estimated to be involved in the metabolism of drugs, chemicals and endogenous substrates, and hepatic CYPs may participate in the pathogenesis of liver diseases (51). Thus, the present results were in accordance with those from previous studies $(47,51)$.

In conclusion, the present study successfully identified significant genes (such as $P L C B 1, C Y P 2 C 8$ and $C Y P 2 B 6$ ) and pathways (including neuroactive ligand-receptor interaction, purine metabolism and metabolism of xenobiotics mediated by CYP), which may be potential biomarkers associated with HCC. The current study greatly improved the understanding of $\mathrm{HCC}$ in a systematic manner and provided potential biomarkers for early detection and novel therapeutic methods.

\section{Acknowledgements}

The authors would like to express their gratitude to all the collaborators that assisted during the writing of the present manuscript. The authors gratefully acknowledge the Jinan Evidence Based Medicine Science-Technology Center (Jinan, China) for their valuable instructions and suggestions on the selection of the microarray expression profiles and the analytical methods employed in the present study.

\section{References}

1. Kaseb AO, Xiao L, Hassan MM, Chae YK, Lee JS, Vauthey JN, Krishnan S, Cheung S, Hassabo HM, Aloia T, et al: Development and validation of a scoring system using insulin-like growth factor to assess hepatic reserve in hepatocellular carcinoma. J Natl Cancer Inst 106: pii: dju088, 2014.

2. Arzumanyan A, Reis HM and Feitelson MA: Pathogenic mechanisms in HBV- and HCV-associated hepatocellular carcinoma. Nat Rev Cancer 13: 123-135, 2013.

3. Aoki T, Kokudo N, Matsuyama Y, Izumi N, Ichida T, Kudo M, $\mathrm{Ku}$ Y, Sakamoto M, Nakashima O, Matsui O, et al: Prognostic impact of spontaneous tumor rupture in patients with hepatocellular carcinoma: An analysis of 1160 cases from a nationwide survey. Ann Surg 259: 532-542, 2014.

4. Llovet JM, Peña CE, Lathia CD, Shan M, Meinhardt G and Bruix J; SHARP Investigators Study Group: Plasma biomarkers as predictors of outcome in patients with advanced hepatocellular carcinoma. Clin Cancer Res 18: 2290-2300, 2012.

5. Doncheva NT, Kacprowski T and Albrecht M: Recent approaches to the prioritization of candidate disease genes. Wiley Interdiscip Rev Syst Biol Med 4: 429-442, 2012.

6. Srihari S and Ragan MA: Systematic tracking of dysregulated modules identifies novel genes in cancer. Bioinformatics 29: $1553-1561,2013$.

7. Liu KQ, Liu ZP, Hao JK, Chen L and Zhao XM: Identifying dysregulated pathways in cancers from pathway interaction networks. BMC Bioinformatics 13: 126, 2012.

8. Zhang J, Lu K, Xiang Y, Islam M, Kotian S, Kais Z, Lee C, Arora M, Liu HW, Parvin JD and Huang K: Weighted frequent gene co-expression network mining to identify genes involved in genome stability. PLoS Comput Biol 8: e1002656, 2012.

9. Magger O, Waldman YY, Ruppin E and Sharan R: Enhancing the prioritization of disease-causing genes through tissue specific protein interaction networks. PLoS Comput Biol 8: e1002690, 2012.

10. Liu Y, Koyutürk M, Barnholtz-Sloan JS and Chance MR: Gene interaction enrichment and network analysis to identify dysregulated pathways and their interactions in complex diseases. BMC Syst Biol 6: 65, 2012.
11. Jensen LJ, Kuhn M, Stark M, Chaffron S, Creevey C, Muller J, Doerks T, Julien P, Roth A, Simonovic M, et al: STRING 8-a global view on proteins and their functional interactions in 630 organisms. Nucleic Acids Res 37 (Database issue): D412-D416, 2009.

12. Roessler S, Jia HL, Budhu A, Forgues M, Ye QH, Lee JS, Thorgeirsson SS, Sun Z, Tang ZY, Qin LX and Wang XW: A unique metastasis gene signature enables prediction of tumor relapse in early-stage hepatocellular carcinoma patients. Cancer Res 70: 10202-10212, 2010.

13. Roessler S, Long EL, Budhu A, Chen Y, Zhao X, Ji J, Walker R, Jia HL, Ye QH, Qin LX, et al: Integrative genomic identification of genes on $8 p$ associated with hepatocellular carcinoma progression and patient survival. Gastroenterology 142: 957-966. e12, 2012.

14. Irizarry RA, Bolstad BM, Collin F, Cope LM, Hobbs B and Speed TP: Summaries of Affymetrix GeneChip probe level data. Nucleic Acids Res 31: e15, 2003.

15. Bolstad BM, Irizarry RA, Astrand Mand Speed TP: A comparison of normalization methods for high density oligonucleotide array data based on variance and bias. Bioinformatics 19: 185-193, 2003.

16. Bolstad B: affy: Built-in processing methods. http://www. bioconductor.org/packages/release/bioc/vignettes/affy/inst/doc/ builtinMethods.pdf. Accessed March 26, 2014.

17. Williams S: Pearson's correlation coefficient. N Z Med J 109: 38 , 1996.

18. Liu G, Wong L and Chua HN: Complex discovery from weighted PPI networks. Bioinformatics 25: 1891-1897, 2009.

19. Srihari S and Leong HW: A survey of computational methods for protein complex prediction from protein interaction networks. J Bioinform Comput Biol 11: 1230002, 2013.

20. Tomita E, Tanaka A and Takahashi H: The worst-case time complexity for generating all maximal cliques and computational experiments. Theor Comput Sci 363: 28-42, 2006.

21. Gabow HN: An efficient implementation of Edmonds' algorithm for maximum matching on graphs. JACM 23: 221-234, 1976.

22. Huang da W, Sherman BT and Lempicki RA: Systematic and integrative analysis of large gene lists using DAVID bioinformatics resources. Nat Protoc 4: 44-57, 2009.

23. Ford G, Xu Z, Gates A, Jiang J and Ford BD: Expression analysis systematic explorer (EASE) analysis reveals differential gene expression in permanent and transient focal stroke rat models. Brain Res 1071: 226-236, 2006.

24. Diboun I, Wernisch L, Orengo CA and Koltzenburg M: Microarray analysis after RNA amplification can detect pronounced differences in gene expression using limma. BMC genomics 7: 252, 2006.

25. Schmittgen TD and Livak KJ: Analyzing real-time PCR data by the comparative C(T) method. Nat Protoc 3: 1101-1108, 2008.

26. Poduri A, Chopra SS, Neilan EG, Elhosary PC, Kurian MA, Meyer E, Barry BJ, Khwaja OS, Salih MA, Stödberg T, et al: Homozygous PLCB1 deletion associated with malignant migrating partial seizures in infancy. Epilepsia 53: e146-e150, 2012.

27. Jia D, Wei L, Guo W, Zha R, Bao M, Chen Z, Zhao Y, Ge C, Zhao F, Chen T, et al: Genome-wide copy number analyses identified novel cancer genes in hepatocellular carcinoma. Hepatology 54: 1227-1236, 2011.

28. Das T, Diamond DL, Yeh M, Hassan S, Bryan JT, Reyes JD and Perkins JD: Molecular signatures of recurrent hepatocellular carcinoma secondary to hepatitis $\mathrm{C}$ virus following liver transplantation. J Transplant 2013: 878297, 2013.

29. Danielson PB: The cytochrome P450 superfamily: biochemistry, evolution and drug metabolism in humans. Curr Drug Metab 3: 561-597, 2002

30. Booth Depaz IM, Toselli F, Wilce PA and Gillam EM: Differential expression of cytochrome P450 enzymes from the CYP2C subfamily in the human brain. Drug Metab Dispos 43: 353-357, 2015.

31. Yu Y, Ping J, Chen H, Jiao L, Zheng S, Han ZG, Hao P and Huang J: A comparative analysis of liver transcriptome suggests divergent liver function among human, mouse and rat. Genomics 96: 281-289, 2010.

32. Hertz DL, Roy S, Jack J, Motsinger-Reif AA, Drobish A, Clark LS, Carey LA, Dees EC and McLeod HL: Genetic heterogeneity beyond CYP2C8*3 does not explain differential sensitivity to paclitaxel-induced neuropathy. Breast Cancer Res Treat 145: 245-254, 2014. 
33. Bergmann TK, Brasch-Andersen C, Gréen H, Mirza MR, Skougaard K, Wihl J, Keldsen N, Damkier P, Peterson C, Vach W and BrØsen K: Impact of ABCB1 variants on neutrophil depression: A pharmacogenomic study of paclitaxel in 92 women with ovarian cancer. Basic Clin Pharmacol Toxicol 110: 199-204, 2012.

34. Bergmann TK, Brasch-Andersen C, Gréen H, Mirza M, Pedersen RS, Nielsen F, Skougaard K, Wihl J, Keldsen N, Damkier P, et al: Impact of CYP2C8 3 on paclitaxel clearance: A population pharmacokinetic and pharmacogenomic study in 93 patients with ovarian cancer. Pharmacogenomics J 11: 113-120, 2011.

35. Läpple F, von Richter O, Fromm MF, Richter T, Thon KP, Wisser H, Griese EU, Eichelbaum M and Kivistö KT: Differential expression and function of CYP2C isoforms in human intestine and liver. Pharmacogenetics 13: 565-575, 2003.

36. Zhang SY, Surapureddi S, Coulter S, Ferguson SS and Goldstein JA: Human CYP2C8 is post-transcriptionally regulated by microRNAs 103 and 107 in human liver. Mol Pharmacol 82: 529-540, 2012

37. Naraharisetti SB, Lin YS, Rieder MJ, Marciante KD, Psaty BM, Thummel KE and Totah RA: Human liver expression of CYP2C8: gender, age, and genotype effects. Drug Metab Dispos 38: 889-893, 2010.

38. Ekins S and Wrighton SA: The role of CYP2B6 in human xenobiotic metabolism. Drug Metab Rev 31: 719-754, 1999.

39. Kumagai J, Fujimura T, Takahashi S, Urano T, Ogushi T, Horie-Inoue K, Ouchi Y, Kitamura T, Muramatsu M, Blumberg B and Inoue S: Cytochrome P450 2B6 is a growth-inhibitory and prognostic factor for prostate cancer. Prostate 67: 1029-1037, 2007.

40. Lee JH, Shim JH, Chung YH, Lee D, Lee HC and Shin ES: Genetic polymorphisms associated with treatment toxicity after sorafenib combination therapy in Korean patients with hepatocellular carcinoma. Clin Mol Hepatol 17: 36, 2011.

41. Chen H, Shen ZY, Xu W, Fan TY, Li J, Lu YF, Cheng ML and Liu J: Expression of P450 and nuclear receptors in normal and end-stage Chinese livers. World J Gastroenterol 20: 8681-8690, 2014.
42. Cillo C, Schiavo G, Cantile M, Bihl MP, Sorrentino P, Carafa V, D'Armiento M, Roncalli M, Sansano S, Vecchione R, et al: The HOX gene network in hepatocellular carcinoma. Int J Cancer 129: 2577-2587, 2011

43. Liu Z, Gartenhaus RB, Tan M, Jiang F and Jiao X: Gene and pathway identification with Lp penalized Bayesian logistic regression. BMC Bioinformatics 9: 412, 2008.

44. Wang L, Huang J, Jiang $M$ and Zheng X: AFP computational secreted network construction and analysis between human hepatocellular carcinoma (HCC) and no-tumor hepatitis/cirrhotic liver tissues. Tumor Biol 31: 417-425, 2010.

45. Zhao Y, Xue F, Sun J, Guo S, Zhang H, Qiu B, Geng J, Gu J, Zhou X, Wang W, et al: Genome-wide methylation profiling of the different stages of hepatitis B virus-related hepatocellular carcinoma development in plasma cell-free DNA reveals potential biomarkers for early detection and high-risk monitoring of hepatocellular carcinoma. Clin Epigenetics 6: 30, 2014.

46. Liao KC, Pu SJ, Lin CH, Chang HJ, Chen YJ and Liu MS: Association between the metabolic syndrome and its components with falls in community-dwelling older adults. Metab Syndr Relat Disord 10:447-451, 2012.

47. Chan W, Lin S, Sun S, Liu H, Luk JM and Cai Z: Metabolomics analysis of the responses to partial hepatectomy in hepatocellular carcinoma patients. Am J Analyt Chem 2: 142-151, 2011.

48. Baniasadi H, Gowda GA, Gu H, Zeng A, Zhuang S, Skill N, Maluccio $M$ and Raftery D: Targeted metabolic profiling of hepatocellular carcinoma and hepatitis C using LC-MS/MS. Electrophoresis 34: 2910-2917, 2013.

49. Cheng S, Prot JM, Leclerc E and Bois FY: Zonation related function and ubiquitination regulation in human hepatocellular carcinoma cells in dynamic vs. static culture conditions. BMC Genomics 13: 54, 2012.

50. Lv J, Zhu B, Zhang L, Xie Q and Zhuo W: Detection and screening of small molecule agents for overcoming Sorafenib resistance of hepatocellular carcinoma: A bioinformatics study. Int J Clin Exp Med 8: 2317-2325, 2015.

51. Villeneuve JP and Pichette V: Cytochrome P450 and liver diseases. Curr Drug Metab 5: 273-282, 2004. 